\title{
Growth of massive cementite layers; thermodynamic parameters and kinetics
}

\author{
Marc Nikolussi · Andreas Leineweber • \\ Eric Jan Mittemeijer
}

Received: 20 June 2008/ Accepted: 3 December 2008/Published online: 27 December 2008

(C) The Author(s) 2008. This article is published with open access at Springerlink.com

\begin{abstract}
Massive, pure cementite layers were grown on ferrite substrates by nitrocarburising in a dedicated $\mathrm{NH}_{3} /$ $\mathrm{H}_{2} / \mathrm{CO} / \mathrm{N}_{2}$ containing gas atmosphere at temperatures in the range of 783-843 K. From the parabolic layer-growth constants, an "apparent" activation energy for cementitelayer growth of $109 \pm 12 \mathrm{~kJ} / \mathrm{mol}$ was obtained. This "apparent" activation energy can be subdivided into a positive contribution due to the activation energy for (tracer) diffusion of carbon in cementite and a negative contribution due to the temperature dependence of the difference of the carbon activity in cementite at the surface and at the interface cementite/ferrite.
\end{abstract}

\section{Introduction}

Cementite, $\mathrm{Fe}_{3} \mathrm{C}$, is, besides ferrite, the major constituent of plain carbon and mildly alloyed steels. Notwithstanding its great technological importance, little is known about the intrinsic properties of cementite. This dearth of fundamental knowledge on cementite is mainly due to the difficulty in preparing single-phase (single- or polycrystalline) cementite specimens. In the past, several works dealt with the production of bulk cementite specimens applying different methods [1-5]. Yet, only recently, it was reported for the first time that massive cementite layers can be grown on ferrite substrates by a dedicated gaseous

M. Nikolussi · A. Leineweber $(\bowtie) \cdot$ E. J. Mittemeijer

Max Planck Institute for Metals Research, Heisenbergstrasse 3,

70569 Stuttgart, Germany

e-mail: a.leineweber@mf.mpg.de nitrocarburising process [6]. The addition of ammonia to the carburising gas mixture was found to be decisive for the suppression of both the formation of graphitic soot and the disintegration of cementite once formed (metal dusting).

Gaseous nitrocarburising of iron or iron-based alloys is a thermochemical heat treatment usually performed at temperatures between 773 and $853 \mathrm{~K}$ below the ternary eutectoid temperature of the $\mathrm{Fe}-\mathrm{N}-\mathrm{C}$ solid solution [7]. By this process, which gathered great technical importance during the last decades $[8,9]$, nitrogen and carbon delivered by the gas phase are diffusionally incorporated into the surface-region of the iron-based workpiece [7].

This results in (i) the formation of a compound layer (several $10-\mu \mathrm{m}$ thick) containing, depending on the treatment temperature (and pressure) and the composition of the gas atmosphere, different phases, typically $\gamma^{\prime}-\mathrm{Fe}_{4} \mathrm{~N}_{1-y}$ and $\varepsilon-\mathrm{Fe}_{3}(\mathrm{~N}, \mathrm{C})_{1+x}[10]$, but sometimes, under certain circumstances, also cementite [6,11], and (ii) the formation of a diffusion zone (several $100-\mu \mathrm{m}$ thick), enriched with interstitial nitrogen (and carbon). Whereas the compound layer is responsible for a considerable enhancement of the corrosion and wear resistance, the diffusion zone enhances the fatigue endurance [12].

The present work focuses on an analysis of cementitelayer growth kinetics. Massive cementite layers were grown on ferrite substrates in the treatment-temperature range of 783-843 $\mathrm{K}$, thus extending the preceding study [6] in which only experiments at $T=823 \mathrm{~K}$ were conducted. The experimental values for the (temperature dependence of the) parabolic growth constant were determined. These results were evaluated in terms of an "apparent" activation energy for cementite-layer growth and discussed with reference to previous work on the kinetics of cementite (layer) growth [4, 13-19]. The discrepancy between the results of the present work and the 
literature data are discussed in terms of thermodynamic consequences of the different gas atmospheres used.

\section{Experimental}

Pure iron foils $(20 \mathrm{~mm} \times 25 \mathrm{~mm})$ were produced by cold rolling a ferrite cast rod (Alfa Aesar, $99.98 \mathrm{wt} \% \mathrm{Fe}$ ) on both sides to a thickness of $1 \mathrm{~mm}$. The specimens to be nitrocarburised were cut from this cold-rolled plate, ground, polished (final stage $1 \mu \mathrm{m}$ diamond suspension) and cleaned ultrasonically in ethanol. Prior to the nitrocarburising process, the specimens were recrystallised at $973 \mathrm{~K}$ for $2 \mathrm{~h}$ under a hydrogen flow of $200 \mathrm{ml} / \mathrm{min}$, polished (final stage $1 \mu \mathrm{m}$ diamond suspension) and cleaned ultrasonically in ethanol.

Gaseous nitrocarburising was carried out in a vertical quartz-tube furnace. To retain solid states produced at the nitrocarburising temperature, the quartz-tube furnace was equipped at its end with a water (flushed with $\mathrm{N}_{2}$ ) container for quenching the specimens to room temperature. The iron foils were suspended on a quartz fibre and positioned in the middle of the furnace where the process temperature prevailed, controlled within $\pm 1 \mathrm{~K}$. In order to quench the specimens to room temperature, the quartz fibre was destroyed by a mechanical device and the specimen dropped into the water container. The gas atmosphere was composed of 13 vol.\% ammonia (99.999 vol.\% purity) as a nitrogenproviding medium, 58 vol.\% hydrogen (99.999 vol.\% purity), 20 vol.\% carbon monoxide (99.97 vol.\% purity) as a carbon-providing medium and 9 vol.\% nitrogen (99.999 vol.\% purity) as inert gas; each gas flux was controlled by a mass-flow controller. In order to ensure that ammonia dissociation, which would change the composition of the gas atmosphere, can be neglected, an overall linear flow rate of $13.5 \mathrm{~mm} / \mathrm{s}$ (calculated for the gas volume at room temperature) through the quartz retort (diameter $28 \mathrm{~mm}$ ) was maintained.

Four sets of nitrocarburising experiments were performed at $T=783,803,823$ and $843 \mathrm{~K}$ (the data for $T=823 \mathrm{~K}$ were already reported in a preceding study [6]) for different treatment times in the range of $5 \mathrm{~min}-48 \mathrm{~h}$.

After gaseous nitrocarburising and quenching, the specimens were cut into two pieces, one used for optical microscopy and one used for X-ray diffraction (XRD).

In order to avoid mechanical damage at the surface of the specimen (parts) used for metallographic analysis and to ensure a sufficient sharpness at the specimen surface in the cross-sections, the specimens were covered with a protective nickel layer electrodeposited at $333 \mathrm{~K}$ using a Watts bath [10, 20]. Thereafter, the specimens were embedded (Polyfast, Buehler $\mathrm{GmbH}$ ), ground, polished (final stage $1 \mu \mathrm{m}$ diamond suspension) and etched using
1 vol. $\%$ Nital containing 0.1 vol. $\% \mathrm{HCl}$ [21]. To improve the optical contrast between the massive cementite layer and the ferrite substrate, the cross-sectional specimens were additionally stained using either an alkaline potassium permanganate solution $(1 \mathrm{~g} \mathrm{NaOH}, 1 \mathrm{~g} \mathrm{KOH}$ and $4 \mathrm{~g}$ $\mathrm{KMnO}_{4}$ per $100 \mathrm{ml}$ distilled water) at $328 \mathrm{~K}[6,22]$ of which the effect is similar to that of a Murakami solution $[10,22]$ or an alkaline sodium picrate solution $(25 \mathrm{~g} \mathrm{NaOH}$ and $2 \mathrm{~g}$ picric acid per $75 \mathrm{ml}$ distilled water) at room temperature [22]. By these procedures, the massive cementite layer gets, due to its high carbon content, severely stained and can therefore easily be distinguished upon light optical microscopy from the ferrite substrate, which remains unstained. Light optical microscopy was performed using a Zeiss Axiophot microscope.

For the determination of the cementite-layer thickness, several micrographs were taken from each specimen. From these micrographs, the area of the massive cementite layer was measured and divided through its lateral length, yielding, after averaging the experimental values obtained from several micrographs, the cementite-layer thickness.

Phase identification was carried out by X-ray diffraction to verify the presence of a massive cementite layer on the ferrite substrate. A PANalytical X'Pert Multi-Purpose Diffractometer equipped with a graphite monochromator in the diffracted beam, utilising $\operatorname{CoK} \alpha$ radiation and BraggBrentano geometry, was used. To achieve better crystallite statistics, the specimens were rotated during the measurements around their surface normal.

\section{Results and evaluation}

All experiments performed led to the formation of massive cementite layers on the ferrite substrates (cf. Fig. 1).

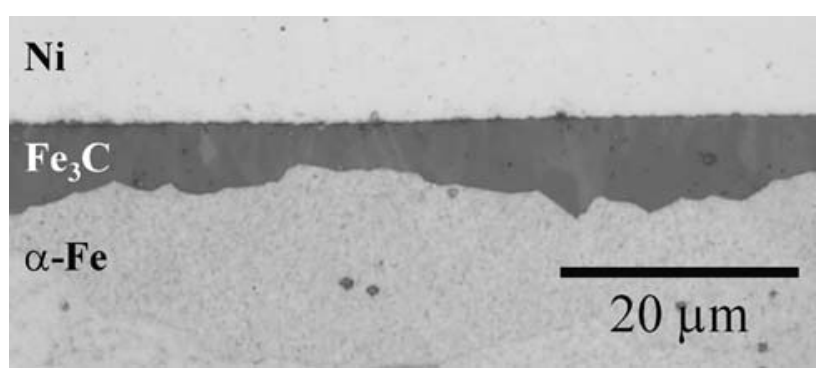

Fig. 1 Light optical microscopic image of a metallographic crosssection after etching with 1 vol.\% Nital containing $1 \mathrm{vol} . \% \mathrm{HCl}$ and staining with an alkaline sodium picrate solution. The massive cementite layer (stained) was grown on the ferrite substrate (unstained) by gaseous nitrocarburising at $823 \mathrm{~K}$ for $48 \mathrm{~h}$ using a gas atmosphere containing $13 \mathrm{vol} \% \mathrm{NH}_{3}, 58$ vol. $\% \mathrm{H}_{2}$ (nitriding potential $r_{\mathrm{N}}=0.3 \mathrm{~atm}^{-1 / 2}$ ), $20 \mathrm{vol} . \% \mathrm{CO}$ and 9 vol. $\% \mathrm{~N}_{2}$ (as inert gas) 


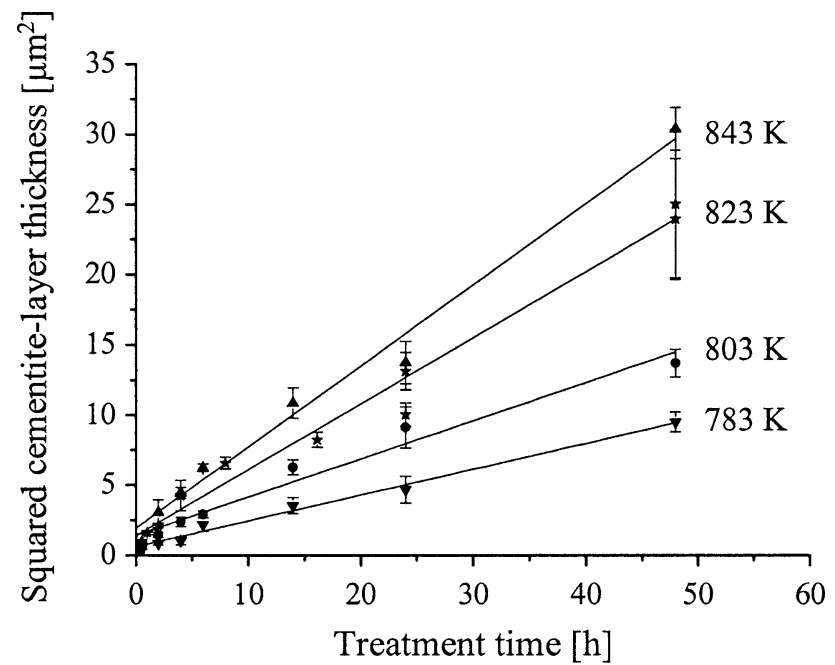

Fig. 2 Squared cementite-layer thickness versus treatment time at $783,803,823$ and $843 \mathrm{~K}$, using a gas atmosphere composed of 13 vol. $\% \mathrm{NH}_{3}, 58$ vol. $\% \mathrm{H}_{2}$ (nitriding potential $r_{\mathrm{N}}=0.3 \mathrm{~atm}^{-1 / 2}$ ), 20 vol.\% $\mathrm{CO}$ and 9 vol.\% $\mathrm{N}_{2}$. The straight lines represent leastsquares fitting of Eq. 1 for the different treatment temperatures to the respective experimental data $(t>1 \mathrm{~h})$ yielding the growth constant $k$ and the hypothetical initial cementite-layer thickness $S_{0}$ (cf. Table 1)

The squared value of the experimentally determined cementite-layer thickness (cf. Sect. Experimental) was plotted versus the treatment time at the various temperatures (cf. Fig. 2). Occurrence of parabolic layer growth from $t=0$ onwards is given by a straight line through the origin of the plot of the squared layer thickness versus time (cf. Fig. 2). Such a simple parabolic layer growth was assumed for treatment times larger than $1 \mathrm{~h}$. Evidently, as follows from the data in Fig. 2, for treatment times $t<1 \mathrm{~h}$ cementite-layer growth occurred faster than indicated by such a parabolic relation. This can be discussed as follows.

At the beginning of the cementite formation, an incompletely closed cementite layer can occur. Hence, carbon can diffuse through ferrite to the (also bottom tip of the) cementite grains growing from the surface (this diffusion through ferrite is much faster than diffusion through cementite), thus bypassing the cementite. Further, shortly after establishment of a closed cementite layer, short-circuit diffusion of carbon may occur through thin and defectrich parts of the cementite layer, exhibiting a relatively high grain-boundary density. Both 'bypass/short-circuit' mechanisms for carbon lead to relatively fast cementite growth as compared to later stages (cf. [6]). The initial 'bypass/short-circuit' mechanism involves that the parabolic cementite-layer growth law pertaining to later stages of cementite-layer growth has to be modified by introducing a hypothetical initial cementite-layer thickness $S_{0}$ $(>0)$ at $t=0$. Hence, the time-dependence of the cementite-layer growth is given by
Table 1 Experimentally determined growth constants $k$ and hypothetical initial cementite-layer thicknesses $S_{0}$ at four different treatment temperatures $T=783,803,823$ and $843 \mathrm{~K}$

\begin{tabular}{lll}
\hline $\begin{array}{l}\text { Treatment } \\
\text { temperature }[\mathrm{K}]\end{array}$ & $\begin{array}{l}\text { Growth constant } \\
{\left[\mathrm{m}^{2} / \mathrm{s}\right]}\end{array}$ & $\begin{array}{l}\text { Initial cementite-layer } \\
\text { thickness }[\mathrm{m}]\end{array}$ \\
\hline 783 & $(5.1 \pm 0.2) \cdot 10^{-17}$ & $(0.79 \pm 0.09) \cdot 10^{-6}$ \\
803 & $(7.6 \pm 0.6) \cdot 10^{-17}$ & $(1.20 \pm 0.14) \cdot 10^{-6}$ \\
823 & $(1.3 \pm 0.7) \cdot 10^{-16}$ & $(1.19 \pm 0.16) \cdot 10^{-6}$ \\
843 & $(1.6 \pm 0.8) \cdot 10^{-16}$ & $(1.39 \pm 0.16) \cdot 10^{-6}$ \\
\hline
\end{tabular}

The results were obtained by least-squares fitting of Eq. 1 to the respective experimental data (cf. Fig. 2)

$S^{2}(t)=k \cdot t+S_{0}^{2} \quad($ for $t>1 \mathrm{~h})$,

where $S$ denotes the cementite-layer thickness, $k$ the growth constant and $t$ the treatment time.

Least-squares fitting of Eq. 1 to the experimental data at the four treatment temperatures for $t>1 \mathrm{~h}$ (cf. Fig. 2) yielded the results for the growth constant and the hypothetical initial cementite-layer thickness shown in Table 1.

An Arrhenius-type temperature dependence is adopted for the growth constant $k$

$k=k_{0} \cdot \exp \left(-\frac{Q_{\text {app }}}{R T}\right)$,

where $k_{0}$ denotes a pre-exponential factor, $Q_{\text {app }}$ an "apparent" activation energy of cementite-layer growth, $R$ the gas constant and $T$ the treatment temperature. Then, a plot of the logarithm of the growth constant versus the inverse treatment temperature should yield a straight line. Such linear dependence is observed indeed (cf. Fig. 3), validating the application of Eq. 2 (see also Sect. Interpretation of the "apparent" activation energy). The slope of

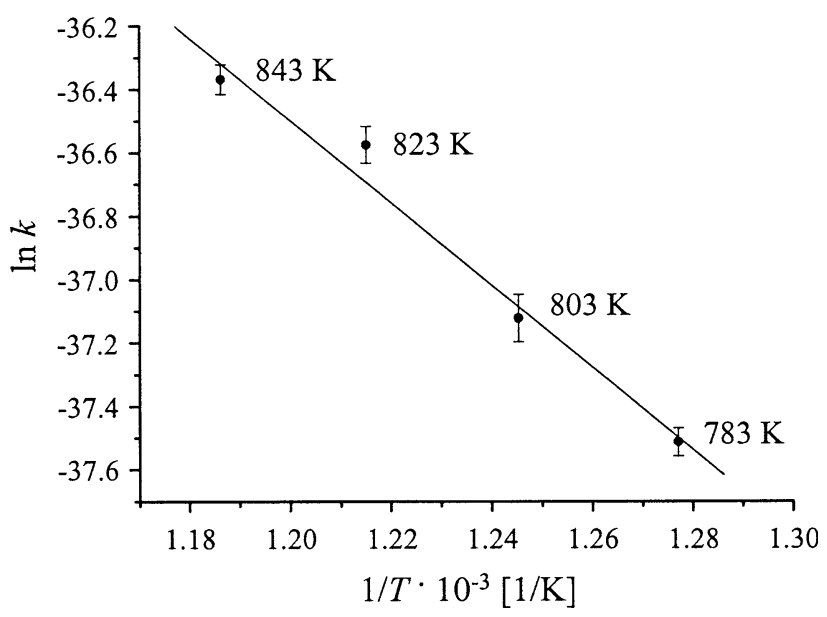

Fig. 3 Arrhenius plot: natural logarithm of the growth constant $k$ versus the inverse treatment temperature 1/T. The "apparent" activation energy of the cementite-layer growth can be calculated from the slope of the straight line which was obtained by least-squares fitting of Eq. 2 to the experimental data 
the plot, $m=-Q_{\text {app }} / R$, yields an "apparent" activation energy equal to $109 \pm 12 \mathrm{~kJ} / \mathrm{mol}$.

\section{Discussion}

Parabolic layer growth for interstitial compounds

In the literature, the growth kinetics of pure iron nitride layers [23-26] but also of cementite layers [4, 15, 16] was described by a parabolic growth law. A comparison of the rates of cementite-layer growth and $\gamma^{\prime}-\mathrm{Fe}_{4} \mathrm{~N}_{1-y}$-layer growth indicates that, at the same temperature, $\gamma^{\prime}-\mathrm{Fe}_{4} \mathrm{~N}_{1-y^{-}}$ layer growth proceeds faster than cementite-layer growth, which can be ascribed to nitrogen volume diffusion in $\gamma^{\prime}-\mathrm{Fe}_{4} \mathrm{~N}_{1-y}$ being faster than carbon volume diffusion in cementite.

Parabolic layer growth according to Eq. 1 with $S_{0}=0$ is expected in particular for close-to-stoichiometric interstitial compounds like $\gamma^{\prime}-\mathrm{Fe}_{4} \mathrm{~N}$ and cementite if the following general assumptions are valid [18, 27]: (a) local equilibrium at the interface gas atmosphere/specimen surface; (b) local equilibrium at the interface compound layer/ferrite substrate; (c) a completely closed, massive compound layer; i.e. nitrogen/carbon mass transport proceeds exclusively via nitrogen/carbon (volume) diffusion through the massive compound layer.

If these conditions hold, the parabolic growth constant is proportional to the product of (i) the (tracer)diffusion coefficient of nitrogen/carbon ${ }^{1}$ in the compound layer, $D^{*}$-diffusion of the metal component (iron) can generally be neglected [29] —and of (ii) a function $f(\Delta a)$ (taken as time independent), which increases monotonously with the difference of the activity of nitrogen/carbon in the solid at the surface, $a^{\text {surface }}$, and the activity of nitrogen/carbon in the solid at the interface layer/substrate, $a^{\text {interface } 2 \text { : }}$

$k \propto D^{*} \cdot f(\Delta a)$

with

$\Delta a=a^{\text {surface }}-a^{\text {interface }}$.

According to the assumption of local equilibrium at the surface (see above), the activity $a^{\text {surface }}$ is determined by the composition of the gas atmosphere, and is controlled as

\footnotetext{
${ }^{1}$ It was shown that, due to cementite layer/ferrite substrate misfitstrain development, specific crystallographic directions of cementite, $[u v w]_{\mathrm{C}}$ with $w$ large, occur parallel to the surface normal of the specimen [28]. The diffusion data for carbon presented in this work pertain to these directions.

${ }^{2}$ For details concerning the dependence of $k$ on the activities $a^{\text {surface }}$ and $a^{\text {interface }}$ with respect to the operating diffusion mechanism (see [18]).
}

described in Sect. Thermodynamics of gaseous nitrocarburising. According to the assumption of local equilibrium at the layer/substrate interface, the activity $a^{\text {interface }}$ is compatible with the corresponding phase boundary in the ternary phase diagram $\mathrm{Fe}-\mathrm{N}-\mathrm{C}$.

Thermodynamics of gaseous nitrocarburising

Upon exposing the ferrite substrates to the $\mathrm{NH}_{3} / \mathrm{H}_{2} / \mathrm{CO} / \mathrm{N}_{2^{-}}$ containing gas atmosphere, $\mathrm{N}$ and $\mathrm{C}$ are introduced into the solid, where $\mathrm{NH}_{3}$ and $\mathrm{CO}$ act as the nitrogen and carbonproviding media. ${ }^{3}$

Since thermal decomposition of $\mathrm{NH}_{3}$ as well as decomposition of (metastable) iron nitrides are slow, ${ }^{4}$ transfer of nitrogen from the gas atmosphere into the specimen can be described by the following heterogeneous chemical equilibrium:

$\mathrm{NH}_{3} \rightleftharpoons[\mathrm{N}]+\frac{3}{2} \mathrm{H}_{2}$,

where $[\mathrm{N}]$ denotes nitrogen either dissolved in the ferrite substrate (solid solution) or in iron nitride. The chemical potential $\mu_{\mathrm{N}}^{\text {surface }}$ and the activity $a_{\mathrm{N}}^{\text {surface }}$ of nitrogen dissolved in the solid at the surface (assumed to be in equilibrium with the gas atmosphere) obey:

$$
\begin{aligned}
\mu_{\mathrm{N}}^{\text {surface }} & =\frac{1}{2} \mu_{\mathrm{N}_{2}}^{0}+R T \ln a_{\mathrm{N}}^{\text {surface }} \\
& =\mu_{\mathrm{NH}_{3}}^{0}-\frac{3}{2} \mu_{\mathrm{H}_{2}}^{0}+R T \ln \left(r_{\mathrm{N}} \cdot\left(p^{0}\right)^{\frac{1}{2}}\right),
\end{aligned}
$$

where $\mu_{\mathrm{N}_{2}}^{0}, \mu_{\mathrm{NH}_{3}}^{0}$ and $\mu_{\mathrm{H}_{2}}^{0}$ are the chemical potentials of nitrogen, ammonia and hydrogen in the gaseous reference state (pressure $p^{0}=1 \mathrm{~atm}$ ) and $r_{\mathrm{N}}=\frac{p_{\mathrm{NH}_{3}}}{p_{\mathrm{H}_{2}}^{3 / 2}}$ denotes the socalled nitriding potential, where $p_{\mathrm{NH}_{3}}$ and $p_{\mathrm{H}_{2}}$ are the partial pressures of ammonia and hydrogen in the gas atmosphere. Hence, according to Eq. 6, the nitriding potential $^{5}$ of the gas atmosphere at the surface fixes the chemical potential and the activity of nitrogen in the solid at the surface [31]. It follows that the activity $a_{\mathrm{N}}^{\text {surface }}$ of nitrogen dissolved in the solid at the surface (assumed to be in equilibrium with the gas atmosphere) obeys:

$a_{\mathrm{N}}^{\text {surface }}=\exp \left(-\frac{\frac{1}{2} \mu_{\mathrm{N}_{2}}^{0}+\frac{3}{2} \mu_{\mathrm{H}_{2}}^{0}-\mu_{\mathrm{NH}_{3}}^{0}}{R T}\right) \cdot\left(p^{0}\right)^{1 / 2} \cdot r_{\mathrm{N}}$.

Transfer of carbon from the gas atmosphere into the specimen is associated with the presence of $\mathrm{CO}$ in the gas

\footnotetext{
3 At atmospheric pressure and at the applied treatment temperatures, $\mathrm{N}_{2}$ does not react with solid iron; it can be regarded as an inert gas.

${ }_{4}^{4}$ This implies that the reaction $\mathrm{NH}_{3} \rightleftharpoons \frac{1}{2} \mathrm{~N}_{2}+{ }_{2}^{3} \mathrm{H}_{2}$ is necessarily not in equilibrium in the gas phase, which is assured by a sufficiently large gas flux though the furnace (cf. Sect. Experimental and Ref. [26]), which is a prerequisite for significant $\mathrm{N}$ transfer to the iron.

${ }^{5}$ In the present experiments, a nitriding potential of $r_{\mathrm{N}}=0.3 \mathrm{~atm}^{-1 / 2}$ was applied (cf. Sect. Experimental) which, in absence of $\mathrm{CO}$, would lead to the formation of a $\gamma^{\prime}-\mathrm{Fe}_{4} \mathrm{~N}_{1-y}$ compound layer [30].
} 
atmosphere and can proceed via the, in principle potently carburising, heterogeneous Boudouard equilibrium:

$2 \mathrm{CO} \rightleftharpoons[\mathrm{C}]+\mathrm{CO}_{2}$,

and the so-called heterogeneous water-gas equilibrium

$\mathrm{CO}+\mathrm{H}_{2} \rightleftharpoons[\mathrm{C}]+\mathrm{H}_{2} \mathrm{O}$,

where $[\mathrm{C}]$ denotes carbon dissolved either in the ferrite substrate (solid solution) or in cementite. The hydrogen necessary for the chemical equilibrium given by Eq. 9 is delivered by the $\mathrm{H}_{2}$-containing gas atmosphere. The chemical potentials $\mu_{\mathrm{C},(8)}^{\text {surface }}$ and $\mu_{\mathrm{C},(9)}^{\text {surface }}$ and the activities $a_{\mathrm{C},(8)}^{\text {surface }}$ and $a_{\mathrm{C},(9)}^{\text {surface }}$ of carbon dissolved in the solid at the surface (assumed to be in equilibrium with the gas atmosphere according to either Eqs. 8 or 9 obey:

$$
\begin{aligned}
\mu_{\mathrm{C},(8)}^{\text {surface }} & =\mu_{\mathrm{C}}^{0}+R T \ln a_{\mathrm{C}}^{\text {surface }} \\
& =2 \mu_{\mathrm{CO}}^{0}-\mu_{\mathrm{CO}_{2}}^{0}+R T \ln \left(r_{\mathrm{C},(8)} \cdot\left(p^{0}\right)^{-1}\right),
\end{aligned}
$$

and

$$
\begin{aligned}
\mu_{\mathrm{C},(9)}^{\text {surface }} & =\mu_{\mathrm{C}}^{0}+R T \ln a_{\mathrm{C}}^{\text {surface }} \\
& =\mu_{\mathrm{CO}}^{0}+\mu_{\mathrm{H}_{2}}^{0}-\mu_{\mathrm{H}_{2} \mathrm{O}}^{0}+R T \ln \left(r_{\mathrm{C},(9)} \cdot\left(p^{0}\right)^{-1}\right),
\end{aligned}
$$

where $\mu_{\mathrm{C}}^{0}$ is the chemical potential of graphite in the reference state (pressure $p^{0}=1 \mathrm{~atm}$ ) and $\mu_{\mathrm{CO}}^{0}, \mu_{\mathrm{CO}_{2}}^{0}, \mu_{\mathrm{H}_{2}}^{0}$ and $\mu_{\mathrm{H}_{2} \mathrm{O}}^{0}$ are the chemical potentials of carbon monoxide, carbon dioxide, hydrogen and water in the gaseous reference state (pressure $p^{0}=1 \mathrm{~atm}$ ) and $r_{\mathrm{C},(8)}=\frac{p_{\mathrm{CO}}^{2}}{p_{\mathrm{CO}_{2}}}$ and $r_{\mathrm{C},(9)}=\frac{p_{\mathrm{CC}} \cdot p_{\mathrm{H}_{2}}}{p_{\mathrm{H}_{2} \mathrm{O}}}$ denote the so-called carburising potentials according to the equilibria given by Eqs. 8 and 9, where $p_{\mathrm{CO}}, p_{\mathrm{CO}_{2}}, p_{\mathrm{H}_{2}}$ and $p_{\mathrm{H}_{2} \mathrm{O}}$ are the partial pressures of carbon monoxide, carbon dioxide, hydrogen and water in the gas atmosphere. The carburising potential fixes, similar to the nitriding potential (see above), the chemical potential and the activity of carbon in the solid at the surface. It follows that the activity $a_{\mathrm{C}, \mathrm{i} \text { ) }}^{\text {surface }}$ of carbon in the solid at the surface (assumed to be in equilibrium with the gas atmosphere) obeys for the equilibria given by Eqs. 8 and $9(i=8,9)$ :

$a_{\mathrm{C},(8)}^{\text {surface }}=\exp \left(-\frac{\mu_{\mathrm{C}}^{0}+\mu_{\mathrm{CO}_{2}}^{0}-2 \mu_{\mathrm{CO}}^{0}}{R T}\right) \cdot\left(p^{0}\right)^{-1} \cdot r_{\mathrm{C},(8)}$

and

$a_{\mathrm{C},(9)}^{\text {surface }}=\exp \left(-\frac{\mu_{\mathrm{C}}^{0}+\mu_{\mathrm{H}_{2} \mathrm{O}}^{0}-\mu_{\mathrm{CO}}^{0}-\mu_{\mathrm{H}_{2}}^{0}}{R T}\right) \cdot\left(p^{0}\right)^{-1} \cdot r_{\mathrm{C},(9)}$.

For each of the equilibria given by Eqs. 8 and 9, a separate carbon activity in the solid at the surface can be calculated. The heterogeneous water-gas equilibrium (Eq. 9) is established much faster than the Boudouard equilibrium (Eq. 8) [32]. Therefore, the heterogeneous water-gas equilibrium (Eq. 9) is considered to control the carburising.
In general, in case of equilibrium with the nitrocarburising gas atmosphere, the carbon activity in the solid at the surface complies with the occurrence of simultaneous equilibrium for all possible reactions in the gas atmosphere. Thus, the equilibria given by Eqs. 14-16, have to be considered as well:

$\mathrm{CO}+\mathrm{H}_{2} \mathrm{O} \rightleftharpoons \mathrm{H}_{2}+\mathrm{CO}_{2}$

$\mathrm{CO}+3 \mathrm{H}_{2} \rightleftharpoons \mathrm{CH}_{4}+\mathrm{H}_{2} \mathrm{O}$

$\mathrm{CH}_{4} \rightleftharpoons[\mathrm{C}]+2 \mathrm{H}_{2}$.

In the present experiments, neither $\mathrm{CO}_{2}$ (cf. Eq. 8) nor $\mathrm{H}_{2} \mathrm{O}$ (cf. Eq. 9) were initially present in the nitrocarburising gas atmosphere $\left(p_{\mathrm{CO}_{2}}=p_{\mathrm{H}_{2} \mathrm{O}}=0\right)$. Hence, the carbon activity according to either Eqs. 12 or 13 equals (hypothetically) infinity.

No soot formation occurred in the present experiments. Further, as discussed in Sect. Parabolic layer growth for interstitial compounds, local equilibrium (or at least a stationary state) at the interface gas atmosphere/specimen surface is a prerequisite for parabolic (cementite) layer growth as observed in this work. It is concluded that in the present experiments a finite carbon activity appears to rule in the solid at the surface. Such a finite, "effective" carbon activity can prevail if e.g. due to the chemical reaction according to Eq. 15, a certain amount of water is produced. The stationary presence of this amount of water induces a specific finite, "effective" carbon activity in the solid at the surface according to the heterogeneous water-gas equilibrium (cf. Eq. 13). The value of this "effective" carbon activity at the surface can be estimated using literature data (see what follows in Sect. Interpretation of the "apparent" activation energy).

It is also imaginable that the water determining the carbon activity according to Eq. 13 is the product of the carbondelivering reaction given in Eq. 9. However, this reaction and thereby water formation can only take place at the specimen surface, where its rate is limited by the carbon uptake due to this Eq. 9, which decreases with increasing time. On the other hand, (i) Eq. 15 can, in principle, occur both at the specimen surface, catalysed by the specimen surface, and in the bulk of the gas atmosphere, and (ii) water formation according to Eq. 15 is time independent. A time-dependent water concentration at the specimen surface (as in case of water formation due to Eq. 9) involves a time-dependent "effective" carbon activity in the solid at the surface, which would lead to a deviation from parabolic growth kinetics, which is not observed (cf. Fig. 2). Thus, a major role of water formation according to Eq. 9 seems unlikely.

Interpretation of the "apparent" activation energy

Values reported for the activation energy of carbon volume diffusion in metal carbides range from about $125 \mathrm{~kJ} / \mathrm{mol}$ up 
to $230 \mathrm{~kJ} / \mathrm{mol}$ [29]; for cementite activation, energy values of $154 \mathrm{~kJ} / \mathrm{mol}$ [18] and 163-185 kJ/mol [13, 14] ${ }^{6}$ have been reported. These values differ considerably with the value of $109 \pm 12 \mathrm{~kJ} / \mathrm{mol}$ determined in the present work for the "apparent" activation energy. This difference arises because in the previous works, e.g. [13, 14], the carbon activities in the solid at the surface and at the interface with the substrate were known and accounted for in the determination of $D_{\mathrm{C}}^{*}$ and its activation energy. In the present work, the value of the carbon activity in the solid at the surface is not known directly (cf. Sect. Thermodynamics of gaseous nitrocarburising), but can be derived indirectly.

The "apparent" activation energy value equal to $109 \pm$ $12 \mathrm{~kJ} / \mathrm{mol}$ was determined by Arrhenius-type analysis (see Fig. 3 in Sect. Results and evaluation). The slope of the straight line in the plot of the logarithm of the growth constant versus the inverse treatment temperature (cf. Fig. 3) depends on the temperature dependences of both $D_{\mathrm{C}}^{*}$ (which is certainly of Arrhenius-type) and $f\left(\Delta a_{\mathrm{C}}\right)$. $f\left(\Delta a_{\mathrm{C}}\right)$ varies under the present experimental conditions strongly as a function of the treatment temperature due to the treatment temperature-dependent "effective" $a_{\mathrm{C}}^{\text {surface }}$ (recognising a practically temperature independent $a_{\mathrm{C}}^{\text {interface; }}$ see below). As an Arrhenius-type temperature dependence has been observed for $k$ (cf. Fig. 3), it is suggested that both $D_{\mathrm{C}}^{*}$ and (also) $f_{\text {eff }}$ obey an Arrhenius-type temperature dependence. Thus, it follows from Eq. 3:

$$
\begin{aligned}
k & \propto D_{0, \mathrm{C}}^{*} \cdot \exp \left(-\frac{Q_{\mathrm{D}}}{\mathrm{RT}}\right) \cdot f_{0, \mathrm{eff}} \cdot \exp \left(-\frac{Q_{\text {eff }}}{\mathrm{RT}}\right) \\
& =D_{0, \mathrm{C}}^{*} \cdot f_{0, \text { eff }} \cdot \exp \left(-\frac{Q_{\mathrm{D}}+Q_{\mathrm{feff}_{\mathrm{eff}}}}{\mathrm{RT}}\right)
\end{aligned}
$$

with $f_{\text {eff }}=f\left(\Delta a_{\mathrm{C}}\right)$, and $D_{0, \mathrm{C}}^{*}$ and $f_{0 \text {,eff }}$ are pre-exponential factors for the (tracer) diffusion coefficient of carbon in cementite and $f_{\text {eff }}$, respectively, and $Q_{\mathrm{D}}$ the activation energy for the (tracer)diffusion of carbon in cementite and $Q_{f_{\text {eff }}}$ the energy describing the temperature dependence of $f_{\text {eff. }}$. The "apparent" activation energy $Q_{\text {app }}$ for the cementite-layer growth is composed of the sum of the activation energy for the (tracer) diffusion of carbon in cementite, $Q_{\mathrm{D}}$, and the energy $Q_{f_{\text {eff }}}$ describing the temperature dependence of $f_{\text {eff }}: Q_{\text {app }}=Q_{\mathrm{D}}+Q_{f_{\text {eff }}}$. The discrepancy between the presently determined $Q_{\mathrm{app}}=109 \pm 12 \mathrm{~kJ} / \mathrm{mol}$ and $Q_{\mathrm{D}}=154 \mathrm{~kJ} / \mathrm{mol}$ [18] and $Q_{\mathrm{D}}=163-185 \mathrm{~kJ} / \mathrm{mol}$ $[13,14]$ implies that $Q_{f_{\text {eff }}}$ must be negative.

\footnotetext{
${ }^{6}$ It should be noted that this data was not determined on the basis of growth-rate dependence on temperature. The (tracer-)diffusion coefficient of carbon in cementite, $D_{\mathrm{C}}^{*}$, was determined for only a single temperature. The activation energy for carbon volume diffusion in cementite was then calculated adopting the Arrhenius equation for the diffusion coefficient of carbon in cementite, $D_{\mathrm{C}}^{*}$, employing two different values for the pre-exponential factor $D_{0, \mathrm{C}}^{*}$ for general, interstitial diffusion within metals from the literature.
}

Adopting a monotonous increase of $f_{\text {eff }}$ with $\Delta a_{\mathrm{C}}$ (cf. Sect. Results and evaluation), a negative value for $Q_{f_{\text {eff }}}$ implies that the carbon activity difference $\Delta a_{\mathrm{C}}$ decreases with increasing temperature under the applied cementitelayer growth conditions. The temperature dependence of $\Delta a_{\mathrm{C}}$ is governed by the temperature dependence of the "effective" carbon activity in the solid at the surface; the influence of the temperature dependence of the carbon activity at the interface cementite layer/ferrite substrate is marginal ( $a_{\mathrm{C}}^{\text {interface }}$ is practically constant ${ }^{7}$ ). Therefore, a decreasing carbon activity difference, $\Delta a_{\mathrm{C}}$, with increasing temperature (see above), involves a decreasing "effective" carbon activity in the solid at the surface with increasing temperature.

As compared to the present work, the available experiments reported in the literature [13-15, 19] were performed at lower treatment temperatures. ${ }^{8}$ Therefore, the present experimental data (growth constants for four different temperatures) can only be compared with these literature data (growth constants for various carbon activities in the solid at the surface) by extrapolation of the present experimental data to these lower temperatures. Therefore, in Fig. 4, the straight line obtained by least-squares fitting of Eq. 2 to the experimental data obtained in this work was extrapolated to lower temperatures (dashed line). In [13, 14] and [15, 19], carbon activities in the solid at the surface were adjusted at various values at 723 and $773 \mathrm{~K}$, and the corresponding $k$ (growth constant) values were determined. These $k$ values, corresponding to specific carbon activities as indicated in the figure, lie below the extrapolated dashed line corresponding to the data of the current work (cf. Fig. 4). In order to determine the ("effective") carbon activity in the solid at the surface for the presently applied gas atmosphere at 723 and $773 \mathrm{~K}$, the literature data pertaining to 723 and $773 \mathrm{~K}$ have been (linearly; $k$ vs. $a_{\mathrm{C}}^{\text {surface }}$ ) extrapolated up to the dashed line derived from the present experiments. This approach led to relatively high "effective" carbon activities prevailing at the surface: $a_{\mathrm{c}}=65$ (at $773 \mathrm{~K}$ ) and $a_{\mathrm{c}}=260$ (at $723 \mathrm{~K}$ ). Hence, for the presently applied constant gas atmosphere, the "effective" carbon activity at the surface decreases with increasing treatment temperature. This result is compatible with the negative value obtained for $Q_{f_{\text {eff }}}$.

\footnotetext{
7 The carbon activity in cementite at the interface cementite layer/ ferrite substrate was calculated according to [33]. It was found that $a_{\mathrm{C}}^{\text {interface }}$ varies as a function of temperature from 2.88 (at $783 \mathrm{~K}$ ) to 2.13 (at $843 \mathrm{~K})$. Hence, $a_{\mathrm{C}}^{\text {interface }}$ can be considered as (nearly) constant.

8 The present authors also performed experiments at lower temperatures (773 and $723 \mathrm{~K}$ ) applying the same gas atmosphere as for the other here-reported experiments. The analysis (XRD and optical microscopy) of the resulting compound layers revealed the presence of surface-adjacent Hägg carbide and graphite, additionally to cementite; thus the layers produced were not single-phase cementite.
} 


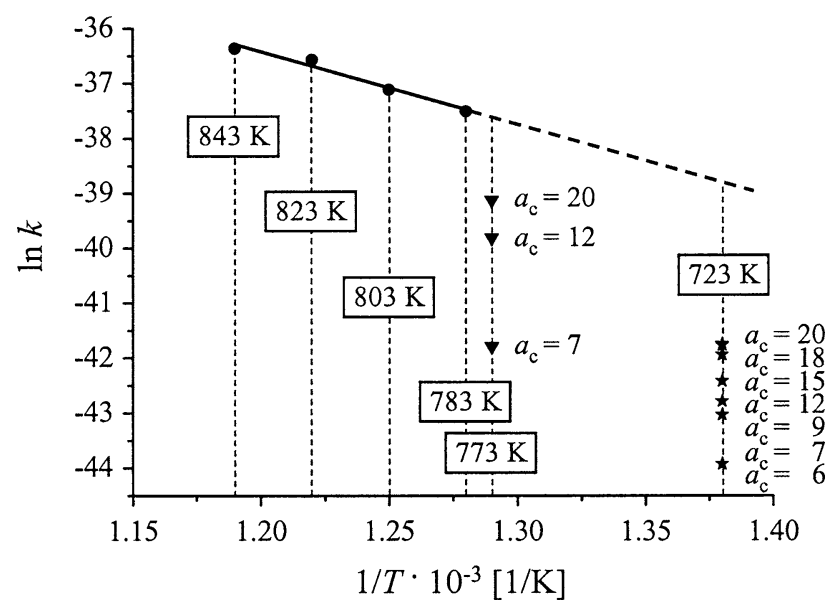

Fig. 4 Extrapolation of the Arrhenius plot shown in Fig. 3: (i) experimental data of the present work have been indicated by dots: experiments performed at $843,823,803$ and $783 \mathrm{~K}$. (ii) Literature data from [19] (triangles): experiments performed at $773 \mathrm{~K}$ at different carbon activities. (iii) Literature data from [13] and [14] (stars): experiments performed at $723 \mathrm{~K}$ at different carbon activities. The straight bold line was obtained by least-squares fitting of Eq. 2 to the experimental data of the present work. The straight dashed line represents the extrapolation of the experimental data of the present work to lower treatment temperatures where the experimental work reported in $[13,14,19]$ was performed

This specific temperature dependence of the "effective" carbon activity in the solid at the surface can be understood as follows. As discussed in Sect. Thermodynamics of gaseous nitrocarburising, the presently applied $\mathrm{NH}_{3} / \mathrm{H}_{2} /$ $\mathrm{CO} / \mathrm{N}_{2}$ containing gas atmosphere is not in thermodynamic equilibrium. Several homogeneous and heterogeneous chemical reactions (equilibria) can occur within the gas atmosphere at the surface of the solid (Eqs. 5, 8, 9, 14-16). In general, chemical equilibria are established faster at elevated temperatures. Hence, it may be expected that more $\mathrm{H}_{2} \mathrm{O}$ is formed (different stationary state) at higher temperatures according to Eq. 15. This leads to an "effective" carbon activity in the solid at the surface which is smaller at higher temperatures.

\section{Conclusions}

(i) Massive, pure cementite layers can be grown on ferrite substrates in the range of $783-843 \mathrm{~K}$ and $5 \mathrm{~min}-48 \mathrm{~h}$ using a gas atmosphere containing 13 vol. $\% \mathrm{NH}_{3}$, 58 vol. $\% \mathrm{H}_{2}, 20$ vol. $\% \mathrm{CO}$ and 9 vol. $\% \mathrm{~N}_{2}$. The addition of ammonia to the gas atmosphere is essential to avoid both soot formation and "metal dusting".

(ii) Cementite-layer growth in the range of 783-843 K obeys a parabolic growth law for $t>1 \mathrm{~h}$. For $t<1 \mathrm{~h}$, 'bypass/short-circuit' mechanisms occur leading to relatively fast cementite growth as compared to later stages $(t>1 \mathrm{~h})$. (iii) The parabolic growth constant exhibits an Arrheniustype temperature dependence. It is proportional to the product of the (tracer-)diffusion coefficient of carbon in the compound layer and the function $f\left(\Delta a_{\mathrm{C}}\right)$ containing the temperature-dependent activity difference of carbon over the thickness of the compound layer. Both factors separately comply with a temperature dependence according to the Arrhenius formalism.

(iv) An "apparent" activation energy, $Q_{\text {app}}$, of $109 \pm$ $12 \mathrm{~kJ} / \mathrm{mol}$ was obtained for cementite-layer growth. This "apparent" activation energy $Q_{\text {app }}$ is composed of a positive contribution $Q_{\mathrm{D}}$ due to the activation energy for (tracer) diffusion of carbon in cementite and a negative contribution $Q_{f_{\text {eff }}}$ due to a decreasing "effective" carbon activity in the solid at the surface with increasing temperature. The formation of a stationary amount of water in the gas atmosphere may induce such a fixed, finite "effective" carbon activity in the solid at the surface.

Open Access This article is distributed under the terms of the Creative Commons Attribution Noncommercial License which permits any noncommercial use, distribution, and reproduction in any medium, provided the original author(s) and source are credited.

\section{References}

1. Toyota Jidoshi KK, Toyota, Aichi, JP (Nonoyama H, Fukuizumi T, Morita A) Japanese Patent P58-122201, 5 July 1983

2. Mizubayashi H, Li SJ, Yumoto H, Shimotomai M (1999) Script Mater 40:773

3. Grabke HJ (2003) Mater Corr 54:736

4. Schneider A, Grabke HJ (2003) Mater Corr 54:793

5. Umemoto M, Todaka Y, Takahashi T, Li P, Tokumiya R, Tsuchiya K (2003) J Metastat Nanocryst Mater 15:607

6. Gressmann T, Nikolussi M, Leineweber A, Mittemeijer EJ (2006) Script Mat 55:723

7. Somers MAJ (2000) Heat Treat Met 27:92

8. Liedtke D, Baudis U, Boßlet J, Huchel U, Klümper-Westkamp H, Lerche W, Spieß H-J (2006) Wärmebehandlung von Eisenwerkstoffen-Nitrieren und Nitrocarburieren. Expert-Verlag, Renningen Malmsheim

9. Unterweiser PM, Gray AG (eds) (1977) Source book on nitriding. ASM, Metals Park, OH

10. Colijn PF, Mittemeijer EJ, Rozendaal HCF (1983) Z Metallkd 74:620

11. Somers MAJ, Mittemeijer EJ (1987) Surf Eng 3:123

12. Mittemeijer EJ (1983) J Heat Treat 3:114

13. Ozturk B, Fearing VL, Ruth JA, Simkovich G (1982) Metall Trans A 13A:1871

14. Ozturk B, Fearing VL, Ruth JA, Simkovich G (1984) Solid State Ionics 12:145

15. Schneider A, Inden G, Grabke HJ (2000) In: Rühle M, Gleiter H (eds) Interface controlled materials (Euromat 99). Weinheim, Wiley-VCH

16. Schneider A (2002) Corr Sci 44:2353

17. Arabczyk W, Konicki W, Narkiewicz U, Jasińska I, Kałucki K (2004) Appl Catal A 266:135 
18. Hillert M, Höglund L, Ågren J (2005) J Appl Phys 98:053511

19. Schneider A, Inden G (2007) Comp Coupl Phase Diagr Thermochem 31:141

20. Chatterjee-Fischer R, Bodenhagen R, Eysell F-W, Hoffmann R, Liedtke D, Mallener H, Rembges W, Schreiner A, Welker G (1995) Wärmebehandlung von Eisenwerkstoffen. Expert-Verlag, Renningen-Malmsheim

21. Wells A (1985) J Mater Sci 20:2439. doi:10:1007/BF00556072

22. Petzow G (1999) Metallographic etching. ASM International, Materials Park, $\mathrm{OH}$

23. Schwerdtfeger K, Grieveson P, Turkdogan ET (1969) Trans TMS AIME 245:2461

24. Somers MAJ, Mittemeijer EJ (1995) Metall Mater Trans A 26A:57
25. Middendorf C, Mader W (2003) Z Metallkd 94:333

26. Mittemeijer EJ, Somers MAJ (1997) Surf Eng 13:483

27. Wagner C (1951) Atom movements. ASM, Cleveland, OH

28. Nikolussi M, Leineweber A, Mittemeijer EJ (2008) Acta Mat 56:5837

29. Wallace TC (1968) In: Hausner H, Bowman MG (eds) Fundamentals of refractory compounds. Plenum Press, New York

30. Lehrer E (1930) Z Elektrochem 36:383

31. Mittemeijer EJ, Slycke JT (1996) Surf Eng 12:152

32. Grabke HJ (1975) Arch Eisenhüttenw 46:75

33. Kunze J (1990) Nitrogen and carbon in iron and steel. AkademieVerlag, Berlin 